livraisons

d'Histoire

de l'Architecture

\section{Livraisons de l'histoire de l'architecture}

26 | 2013

Les ministres et les arts

\title{
L'élaboration du principe d'inaliénabilité pour les collections muséales et les biens du domaine public mobilier sous la Révolution française
}

The building of the non-transferability principle of the museum collections and the goods of the public domain furniture

Die Ausarbeitung des Prinzips der Unveräußerlichkeit musealer Sammlungen und staatlichen Mobiliars zur Zeit der Französischen Revolution

\section{Olivia Brissaud}

\section{OpenEdition}

Journals

Édition électronique

URL : http://journals.openedition.org/lha/343

DOI : 10.4000/lha.343

ISSN : $1960-5994$

Éditeur

Association Livraisons d'histoire de l'architecture - LHA

Édition imprimée

Date de publication : 10 décembre 2013

Pagination : 141-156

ISSN : 1627-4970

Référence électronique

Olivia Brissaud, «L'élaboration du principe d'inaliénabilité pour les collections muséales et les biens du domaine public mobilier sous la Révolution française », Livraisons de l'histoire de l'architecture [En ligne], 26 | 2013, mis en ligne le 10 décembre 2015, consulté le 30 avril 2019. URL : http:// journals.openedition.org/lha/343; DOI : 10.4000/lha.343 


\section{L'ÉLABORATION DU PRINCIPE D'INALIÉNABILITÉ POUR LES COLLECTIONS MUSÉALES ET LES BIENS DU DOMAINE PUBLIC MOBILIER SOUS LA RÉVOLUTION FRANÇAISE}

Le 10 août 1841, un arrêt de la Cour de Cassation rappelle que les objets mobiliers du domaine de la Couronne sont "inaliénables et imprescriptibles de leur nature", et que c'est une "maxime fondamentale en France " qui remonte «à l'ancienne monarchie ${ }^{1}$ ». De nos jours, il est convenu d'établir en France que la notion d'inaliénabilité, principe selon lequel les biens d'État participant du domaine public ne peuvent être vendus, donnés, ni prêtés sans clauses réversibles, était déjà mis en application sous l'Ancien Régime ${ }^{2}$. On excepte cependant la période de la Révolution française qui aurait dérogé à ce principe en permettant l'aliénation des biens du domaine de la Nation par le Code domanial de 1790, décision suivie de nombreuses ventes dont la validité fut confirmée par la suite sous la Restauration ${ }^{3}$ et par divers cas de jurisprudence au XIX ${ }^{\mathrm{e}}$ siècle. Pourtant, si le terme même d'inaliénabilité appliqué aux biens artistiques disparait dans le tumulte révolutionnaire et ne réapparaît qu'avec le sénatus-consulte de 1810 sous l'Empire, c'est justement au cours de ces années révolutionnaires que l'État va peu à peu édifier les fondements juridiques de ce principe, d'autant plus que l'œuvre d'art, contenu de sa valeur intrinsèque et des enjeux mercantiles qu'elle implique, est devenue l'objet d'une réelle législation de la part du gouvernement révolutionnaire.

Si un grand nombre de biens nationalisés, anciennes possessions du clergé, de la Couronne et des émigrés, a été livré au marché de l'art lors de la mise en commerce du patrimoine artistique engagée sous la Révolution française, l'État, conseillé par des marchands et experts familiers du marché de l'art, a su dans la grande redistribution entrainée par ces nationalisations, se constituer une collection d'œuvres d'art d'un mérite considérable et qui correspond parfaitement aux goûts de la fin du XVIII siècle. Dans cet ensemble artistique premièrement composé à partir des

1. D. 1841 I, 332 et S. 1841 I, 742, cité par François Reymond, Le Domaine public mobilier, R.D.P, 1960 , p. 52.

2. "L'inaliénabilité, remonte à l'Ancien Régime. Il explique largement la constitution et la richesse actuelle des collections publiques françaises, qui sont uniques au monde ": discours de Christine Albanel. "Remise du rapport de Jacques Rigaud : réflexion sur la possibilité pour les opérateurs publics d'aliéner des œuvres de leurs collections ", mercredi 7 février 2008.

3. L'irrévocabilité des ventes de biens nationaux fut en effet garantie par le sénatus-consulte de l'an XII, la Constitution du 22 frimaire an VIII ainsi que la Charte de 1814. Voir Jean Dufau, Le Domaine public, Paris, éd. du Moniteur, 1987, p. 227. 
objets sauvés du vandalisme et de la destruction, les révolutionnaires ont distingué deux catégories de biens : ceux qui réclament une protection comme propriété de la Nation, mais simplement parce que l'État veut pouvoir les céder ensuite à leur juste valeur, et les œuvres élues pour participer au nouveau patrimoine artistique. Les objets du premier ensemble s'identifient aux res in patrimonio nostro, décrétées aliénables par le code de 1790 et dont l'État peut disposer librement, considérant qu'il en a la pleine et entière propriété. Si les conservateurs du Muséum cherchent à préserver des dégradations " cinq grandes glaces destinées à des présens que doit faire la Nation ", qu'ils qualifient de "propriété nationale ", il est certain qu'elles ne participent pas d'un patrimoine inaliénable. On les différencie d'une autre catégorie d'objets d'art qui, pour leur esthétisme particulier et leur valeur historique et pédagogique, sont progressivement dotés d'un régime de propriété à part qui implique, en plus d'une protection, l'impossibilité d'être acquis par une personne privée ou une collectivité étrangère à la Nation.

Les artistes, commissaires artistiques et hommes d'État se posent en effet la question de la place des biens artistiques et culturels au sein de l'espace public devenu national. La préservation des biens meubles à cette époque est particulièrement complexe, d'une part les objets d'art peuvent être aisément subtilisés pour être revendus par la suite, d'autre part le concept même d'une limitation de commerce de l'objet meuble est contraire à l'ancien droit qui primait la liberté du commerce. Sous l'Ancien Régime, les biens meubles étaient facilement cessibles et contraints par très peu d'interdits, l'État révolutionnaire peut-il désormais élaborer une protection juridique de l'œuvre d'art en la soustrayant aux lois de la propriété privée ? Au début, c'est l'affaire de nombreuses mesures d'exception prises par les autorités révolutionnaires, mais on peut attester que les biens artistiques sont pleinement considérés par les agents de l'État qui, s'ils donnent la priorité aux pièces des Beaux-Arts, s'attardent également sur certains meubles, productions en matières précieuses, et petits objets à caractère scientifique ou ethnographique. Prenant peu à peu conscience de l'importance de cette question, le gouvernement révolutionnaire va en effet s'efforcer d'accorder une protection particulière à l'objet d'art, bien national, en lui donnant une nouvelle affectation et en assurant sa publicité dans l'espace révolutionnaire : l'œuvre d'art n'est plus un simple ornement, mais un outil qui participe à l'éducation artistique de tous les citoyens, correspondant à une nécessité publique, il devient susceptible d'un usus publicus. Ce sera alors au pouvoir législatif de juger de cette affectation et de déterminer la protection des propriétés nationales: "Les œuvres d'art appartiennent au législateur, il doit les conserver à leur noble destination " ${ }^{5}$, écrit Georges-Marie Raymond dans son Essai sur la peinture et son influence sur les mœurs paru en 1799.

4. Y. Cantarel-Besson, La Naissance du Musée du Louvre. Procès-verbaux des séances du Conservatoire du Muséum national des arts, Paris, éd. de la Réunion des musées nationaux, 1981, t. I, p. 230, séance du 23 fructidor an III (9 septembre 1795).

5. Georges-Marie Raymond, De la Peinture considérée dans ses effets sur les hommes en général et de son influence sur les mours et le gouvernement des peuples, Paris, Charles Pougens, an VII, p. 237. 
La première constatation de cette redistribution patrimoniale est la prise de conscience par les citoyens français d'une propriété collective des œuvres d'art qu'ils ont pour devoir de protéger et qui conduit à la mise en place par les agents du gouvernement d'une administration de l'art. La création des commissions artistiques et de plusieurs institutions publiques entraîne alors de nouvelles mesures à l'égard des œuvres muséales et des biens entrés dans ce qu'on pourrait appeler "le domaine mobilier ". Mais surtout, cette nouvelle considération qui entourent les biens artistiques et culturels a conduit à se poser la question du statut propre de l'objet d'art et permis de préparer son acceptation dans la propriété publique et, par-là, son inaliénabilité et son inviolabilité.

\section{La reconnaissance d'une propriété collective}

Les premières initiatives sont donc de faire reconnaître la propriété publique et collective de ces ouvres d'art. Avec l'institution de la République, l'identité du peuple s'impose et affirme ses droits à participer à la puissance publique. En réaction au vandalisme et aux aliénations, les mesures prises par l'administration culturelle, et, en particulier, la diffusion dans les provinces des instructions pour la conservation des œuvres d'art, permettent aux citoyens de réaliser l'ampleur des richesses qu'ils possèdent et dont ils doivent être les gardiens. Si les "monuments appartiennent à la Nation", c'est qu'ils sont la propriété de tous ses citoyens comme l'explique Félix Vicq d'Azyr, en mars 1794, dans l'Instruction sur la manière d'inventorier et de conserver. Puisque désormais "tous les objets qu'on tenait loin du peuple, ou qu'on ne lui montrait que pour le frapper d'étonnement et de respect, [...] lui appartiennent ${ }^{6}$, celui-ci doit en accepter la charge afin d'en profiter pleinement. Les autorités cherchent donc à donner au peuple la responsabilité des biens de l'art dont ils sont les usufruitiers, mais aussi à montrer la finalité publique de ces " monuments de l'art ".

Si les abus sont multiples et l'indifférence fréquente, on peut attester d'une réelle prise de conscience par un certain public de la destinée des propriétés nationales. En 1797, l'affaire du mausolée du maréchal de Créqui (ill. 1), qu'Alexandre Lenoir cherche à recouvrer pour son Musée des monuments français, montre la distance prise par les citoyens vis-à-vis des acquisitions faites lors des ventes nationales. Le directeur du dépôt des Petits-Augustins propose au gouvernement la récupération d'un monument au duc de Créqui, "à la fois intéressant pour l'histoire et l'histoire de l'art " ${ }^{7}$, acquis lors d'une vente publique par le citoyen

6. B. Deloche et J.-M. Leniaud, La Culture des sans-culottes. Le premier dossier du patrimoine, 17891798, Paris, éd. de Paris, 1989, p. 177.

7. "Monument composé de quatre figures de grandeur naturelle en marbre blanc, exécuté par Hurtrelle et Mazeline, érigé à Charles de Créqui, maréchal de France, en 1622, qui fut tué d'un coup de canon au siège de Brème en 1638, en faisant préparer des batteries ": Archives du musée des Monuments frangais, Inventaire général des richesses d'art de la France, Paris, E. Plon, Nourrit et $\mathrm{C}^{\text {ie }}, 1883-1897$, t. I, p. 75-76, "Lettre de Lenoir au ministre de l'intérieur ", le 15 floréal an V (4 mai 1797). 


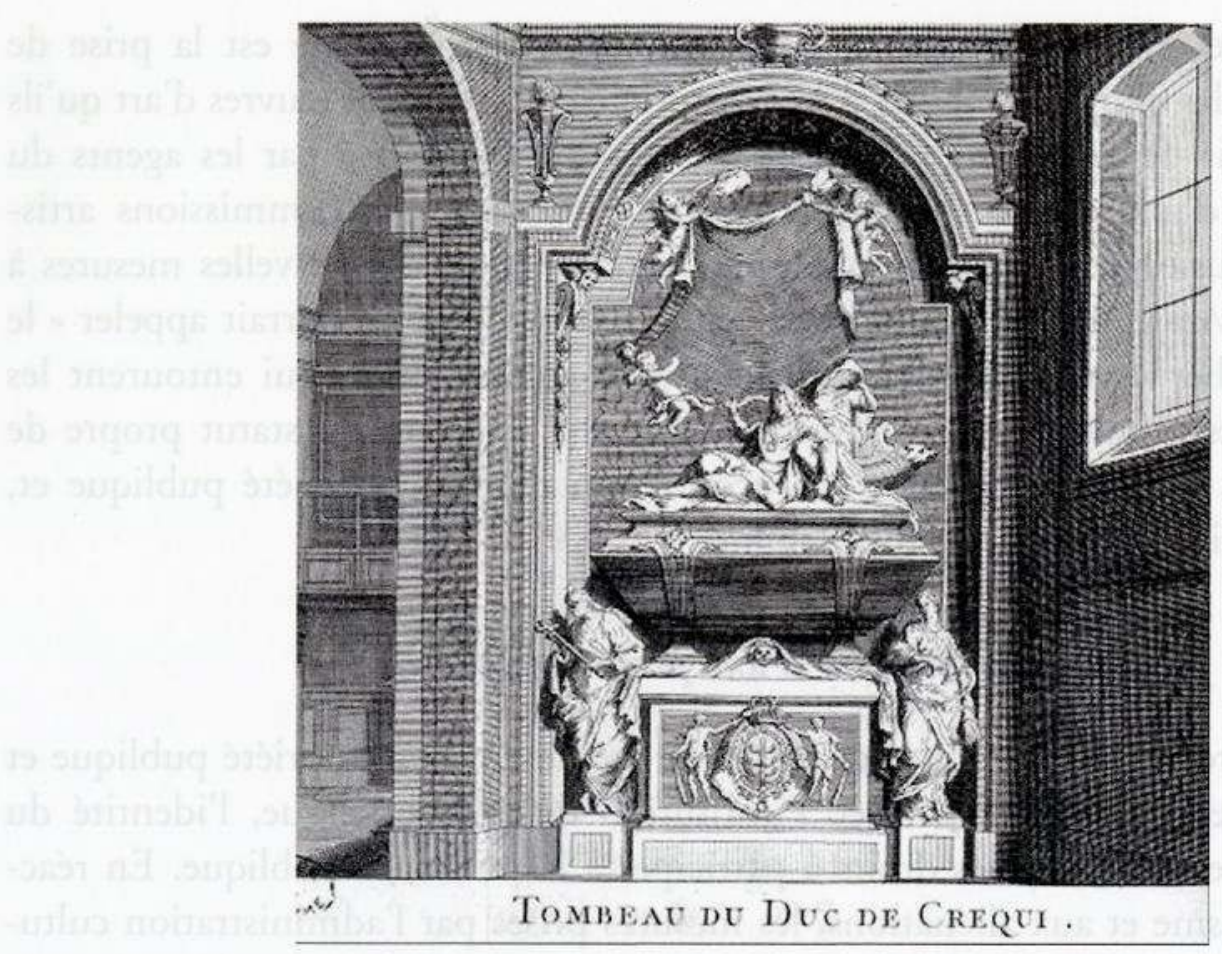

Ill. 1: Le "Tombeau du Duc de Crequi ", gravure de Jean-Baptiste Scotin, taille douce, H. $32 \mathrm{~cm}$, L. $14,5 \mathrm{~cm}$, provenant de la Description de Paris et de ses environs de Jean Aimar Piganiol de la Force, 1742, vol. 2, p. 455 (BnF. Réserve A 200 200).

Marchal. Ce dernier, "sculpteur-marbrier », estime que «si ceux commis par le ministre à la recherche des monuments [avaient] fait leur devoir dans les temps, [il] n'eu[t] jamais été acquéreur ${ }^{8}$ ». S'il demande une juste contrepartie à la cession de ce mausolée, il admet tout de même qu'il n'en est point le propriétaire « légitime " et que la vente n'aurait jamais dû avoir lieu :

On m’a répété mille fois que c'étoit ma propriété. Eh bien, j’avance qu'elle n'est pas la mienne, mais bien celle de la République, par rapport à la conséquence de l'objet, et que je ne dois être considéré que comme dépositaire, à qui il appartient une juste indemnité à cause de l'achat que j'ai fait en deuxième lieu, et qui n'eut jamais, [...] dû se faire. J'ai refusé de vendre plus avantageusement ce monument [...] : c'étoit pour qu'il ne soit pas perdu à l'histoire?'

En tant que simple dépositaire, il se refuse le droit de disposer de ce bien digne d'appartenir à la Nation. Les monuments des arts qui intéressent l'histoire ou qui sont estimés d'une beauté exceptionnelle, auraient donc, par leur nature même, un droit à la propriété publique. Les comités révolutionnaires dotent en effet les Monuments historiques et les œuvres d'art d'une valeur nationale prépondérante

8. Archives du musée des Monuments français, t. I, p. 103, "Lettre du citoyen Marchal au citoyen Ginguené », le 10 nivôse an VI (30 décembre 1797).

9. Ibid. 
en leur attribuant le rôle de témoins du passé et d'instruments éducatifs. La représentation historique en cette époque révolutionnaire est certes la valeur qui prime, mais elle n'exclut pas pour autant les autres. Le citoyen Picault, membre de la Commission du Muséum, plaide pour un droit de la Nation sur le beau. Dans un rapport auprès de la Commission, il s'attarde ainsi sur la beauté de vases étrusques déposés à la Manufacture de Sèvres et demandés par le Muséum. Il justifie la requête du Louvre qui " réclame ces vases parce qu'ils sont beaux et que ce qui est beau dans les arts appartient à ceux qui les cultivent et doit être tiré des dépôts particuliers ". Le commissaire insiste d'ailleurs sur le fait que ces vases doivent être considérés pleinement comme des objets d'art et que, "comme tels, ils doivent orner le Muséum " ${ }^{10}$. La beauté doit donc être "publique " ${ }^{11}$, elle est devenue un attribut du nouveau patrimoine et ne doit plus être confinée pour la jouissance d'une élite.

Pour préserver les biens dispersés, l'État cherche en effet à établir une nouvelle juridiction qui lui permet de défendre sa propriété et de réserver les œuvres à la jouissance de tous. Institution gardienne de ces nouvelles richesses nationales, la Commission des arts refuse ainsi l'échange proposé par le curé de Saint-Germaindes-Prés, Roussineau, en septembre 1793. Ce dernier dit avoir "reçu [...] la promesse de plusieurs des membres de la Commission et de la municipalité ellemême " de recevoir divers tableaux en échange des dix-sept qu'il avait cédés au citoyen Lenoir. Il réclame donc plusieurs sujets de dévotion conservés au Muséum parmi lesquels deux tableaux de Philippe de Champaigne, un "crucifiement de J.C. " et " un Jésus couronné d'épines et ensanglanté ", une Assomption de Valentin de Boulogne et deux petits tableaux de forme ovale dus à Pierre Mignard. Il se base sur le sujet de ces tableaux pour soutenir que "leur place paraît naturellement devoir avoir lieu dans les temples consacrés à la religion et réservés par la loi » ${ }^{12}$. Pourtant, la Commission des monuments prévient le ministre de l'intérieur " qu'il n'est point en son pouvoir de disposer, même par échange, d'aucun des objets qu'elle a fait recueillir et rassembler dans les dépôts nationaux ", ajoutant que "ce serait [...] contrevenir [...] aux lois du 18 octobre et 27 juillet dernier ${ }^{13}$. Les commissaires ont conscience de ne pouvoir céder aussi librement les propriétés de l'État. Le ministre de l'intérieur insiste d'ailleurs auprès de Roussineau sur le nouveau statut acquis par ces œuvres nationalisées:

Toutes les richesses de ce genre sont nationales, et il ne m'est pas permis d'en disposer autrement que pour les réunir dans les dépôts à ce destinés, et servis d'aliment aux muséums, que la gloire des arts et l'intérêt de

10. Arch. nat., $F^{17} 1265$, « Rapport du Citoyen Picault sur des vases étrusques de la manufacture de Sèvres ".

11. Idem.

12. Arch. nat., $\mathrm{F}^{17}$ 1039/B, "Lettre de Roussineau, curé de Saint-Germain-des-Prés à la Commission des arts ", le 12 septembre 1793 .

13. Arch. nat., $\mathrm{F}^{17}$ 1039/B, "Lettre de la Commission des monuments au ministre de l'intérieur, Paré », le 18 septembre 1793. 
l'Instruction publique fait former par la suitte $[s i c]$ dans les département de la République ${ }^{14}$.

Les chefs-d'œuvre de l'art doivent donc être acquis pour la Nation, préservés et surtout exposés au public. Ce public à qui l'on présente les œuvres est en effet un élément déterminant pour l'établissement d'un usus publicus qui existerait dès lors qu'une chose sert "aux besoins d'une universalité indéfinie d'ayants droit, de telle sorte que tous puissent en jouir [...] et que chacun en tire profit directement par lui-même et sans intermédiaire " 15 . On peut donc soutenir l'idée d'une propriété de la Nation sur les œuvres d'art, reconnue par les citoyens, et défendue par les nouvelles instances de l'État. À défaut de res publicae, indisponibles et insusceptibles de propriété, les biens artistiques deviennent des res communes auxquelles tout le monde doit pouvoir accéder. Tout citoyen, même propriétaire privé, s'il a acquis une œuvre qui a le potentiel d'une production nationale, doit donc se sentir concerner et veiller sur ce patrimoine. Les res privatae se meuvent en res publicae au nom de l'intérêt général, le droit révolutionnaire donnant la primauté à la communauté face au particulier.

\section{La mise en place d'une administration et l'élaboration d'une juridiction}

Dès les premières nationalisations et la mise en place d'une administration culturelle, les révolutionnaires définissent un droit de protection et de garde qui découle de la propriété publique. On sait que la notion de collection publique existe déjà avant la Révolution ${ }^{16}$, il suffit de lire les Réflexions de Lafont de SaintYenne $^{17}$ qui, dès 1747 , définit la notion de patrimoine artistique national et établit le rôle du pouvoir dans la conservation des objets. Cependant, la mise en exergue du sentiment de nationalité sous la Révolution fortifie cette idée de biens communs. En 1797, un article du journal de Paris, rédigé lors des «entrées triomphales " des objets d'art au Louvre, montre que les esprits ont pleinement intégré le rôle désormais dévolu aux citoyens français, détenteurs de ces richesses artistiques: les œuvres sont désormais "sous la garde de tous les citoyens qui, ayant intérêt à les conserver, voudront s'en assurer la propriété durable ${ }^{18}$ ".

Pour affirmer les décisions de l'État, les représentants commencent par prendre des lois coercitives contre ceux qui s'en prennent au patrimoine artistique. Des

14. Arch. nat., $\mathrm{F}^{17}$ 1039/B, "Le ministre de l'intérieur au curé Roussineau de Saint-Germain-desPrés ", le 22 septembre 1793.

15. Raymond Saleilles, op. cit., p. 485.

16. Voir Jacques Guillerme, "La naissance au XVIII ${ }^{e}$ siècle du sentiment de responsabilité collective dans la conservation ", Gazette des beaux-arts, 1965, n LXV, p. 155-160.

17. Étienne de Lafont de Saint-Yenne, Réflexions sur quelques causes de l'état présent de la peinture en France, La Haye, Chez Jean Neaulme, 1747, 12 p.

18. Article du Journal de Paris du 5 frimaire an VI (25 novembre 1797), $n^{\circ} 65$, p. 265, dans Édouard Pommier, L'Art de la liberté, Paris, Gallimard, 1991, p. 144. 
mesures de rétorsion sont rapidement prises contre les vandales qui détériorent les propriétés nationales. Le 13 avril 1793, l'Assemblée décrète que « ceux qui seront convaincus d'avoir mutilé [ou] cassé les chefs-d'œuvre en sculpture dans le jardin des Tuileries et autres lieux publics appartenant à la République seront punis de deux années de détention " ${ }^{19}$. Le 6 juin 1793, le savant Lakanal, au nom du Comité d'Instruction publique, demande d'étendre cette peine à quiconque " dégradera les monuments des arts dépendant des propriétés nationales", soulignant qu'il est temps que "la Convention arrête ces funestes excès " ${ }^{20}$. Le commissaire précise que ces propriétés nationales "appartiennent à tous les citoyens en général ", mais qu' " elles ne sont à aucun d'eux en particulier " ${ }^{21}$. La propriété collective implique donc le respect de chacun pour l'intérêt général.

Les commissaires essaient aussi de prendre divers arrêtés pour limiter la cession ou la vente des objets d'art participant du domaine national et le gouvernement se donne les moyens de récupérer les objets d'art qui ont pu lui échapper. L'État met rapidement en place un fond dévolu au ministre de l'intérieur afin de pouvoir acquérir des œuvres, d'empêcher parfois leur aliénation et de les racheter si elles sont passées dans les mains d'un prestataire privé : si un fonds annuel de 300000 livres a été adjugé au ministre de l'intérieur dès le 9 septembre 1791, il est décrété en juillet 1793, d'adjoindre une somme de 1000 livres par an "pour faire acheter dans les ventes particulières les tableaux ou statues qu'il importera à la République de ne pas laisser passer en pays étrangers $»^{22}$. Les instances de l'art comptent fortement sur l'établissement de ce fonds, prévoyant désormais que «nul objet, nulle suite, nulle collection, dignes d'appartenir à la République, ne lui seroient enlevés parce qu'on en négocieroit l'acquisition d'avance et de gré à gré ${ }^{23}$. On peut alors envisager un contrôle a posteriori des ventes, l'État se réservant le droit de racheter certains biens qui lui auraient échappé. En.août 1793, le Comité d'Instruction publique demande ainsi le rachat d'un "globe de seize pouces de diamètre vendu à la vente de Montmorin ", estimé propriété nationale. Il est alors arrêté que le globe «sera retiré des mains de l'acquéreur en lui restituant la somme qu'il justifiera avoir payée pour son acquisition ${ }^{24}$. Une fois la vente conclue, l'administration est avertie du contrat passé et peut donc tenter d'annuler la vente. Aux administrateurs du district de Junien, qui ont constaté la vente "à vil prix " de "différents tableaux et gravures provenant d'émigrés ", et demandent "si la loi [les] autorise à revendiquer ces objets ", les commissaires répondent qu'ils peuvent pro-

19. Archives parlementaires, vol. 62, p. 378, séance du 13 avril 1793.

20. Archives parlementaires, vol. 66, p. 98, "Rapport de Lakanal sur les monuments des arts ", séance du 6 juin 1793.

21. Idem.

22. Archives parlementaires, vol. 69, p. 581, séance du 27 juillet 1793.

23. Arch. nat., $\mathrm{F}^{17} 1238$, "Rapport de la Commission temporaire des arts relatif aux pertes qui résultent pour la République des achats que font les étrangers d'objets précieux et les moyens proposés d'y remédier ".

24. J. Guillaume, Procès-verbaux du Comité d'Instruction publique, t. V, p. 524, séance du 24 thermidor an III (11 août 1793). 
céder à la réclamation "si la vente est postérieure à la loi " ${ }^{25}$. Désormais, l'État peut donc se défendre d'un réel droit de préemption. Le rôle du pouvoir législatif est donc déterminant dans l'établissement de cette nouvelle propriété publique sur les œuvres d'art. Il donne à l'État de nombreux privilèges qui lui permettent d'agir sur le marché de l'art avec bien plus de pouvoir qu'un simple propriétaire privé.

Invoquant les obligations du droit de garde, désormais dévolu à l'administration de l'art, les commissaires et administrateurs rejettent donc les propositions de certains particuliers qui souhaitent acquérir des œuvres d'art nationales : les objets d'art qui ont intégré une entité muséale, et plus encore la prestigieuse institution du Louvre, se voient ainsi attribuer un quasi statut d'immutabilité au sein d'un espace public de l'art. Quand le ministre de la marine et des colonies demande à l'administration du Muséum de lui "faire passer une note des tableaux de marine, tels que les ports de Vernet et autres, dont elle pourrait disposer ", et cela "pour l'arrangement de la grande galerie du Garde-meuble adjointe à la maison de son ministère ", le Conservatoire lui répond que l'administration " ne peut en aucun cas disposer des monuments d'arts, confiés à ses soins ", mais " qu'elle est chargée de leur conservation ". Les productions des Beaux-Arts, même celles d'artistes récents, sont donc empreintes d'une certaine limite dans leur mobilité. La Commission du Muséum conclut d'ailleurs en disant que "cette collection ne peut être distraite des Musées ", et que ces tableaux considérés comme "Monuments historiques de l'art " seront très prochainement "envoyés au Muséum de l'École française à Versailles ${ }^{26}$ ". Ces œuvres d'art sont ainsi refusées à l'agrément d'un agent du gouvernement, ministre qui plus est.

Il semblerait donc que les œuvres de mérite estimées propriétés nationales soient explicitement réservées à l'institution muséale et à son public, mais qu'en est-il du statut des biens mobiliers considérés à la fois comme meubles usagers et décorations qui peuplent désormais les établissements du nouveau gouvernement ${ }^{27}$, ainsi que les habitations "privées" des représentants de l'État? En 1795, avec l'installation du palais directorial au Luxembourg qui devient la résidence des nouveaux membres du gouvernement, et l'aménagement des nouveaux ministères, les envois du Garde-meuble, désormais sous les ordres du ministre des finances, se multiplient pour meubler et orner les habitats ${ }^{28}$. Outre les meubles meublants et objets que l'on pourrait qualifier d'utiles, les intérieurs et extérieurs publics se voient ornés de nombreuses productions des Beaux-Arts, sculptures ou tableaux, comme le révèle le registre de Nesle qui a répertorié les envois destinés aux administrations

25. L. Tuetey, Procès-verbaux de la Commission temporaire des arts, t. II, p. 44, séance du 20 nivôse an III (9 janvier 1795).

26. Y. Cantarel-Besson, op. cit., p. 73, séance du 13 floréal an V (2 mai 1797).

27. "Le Garde-Meuble national est devenu le point central de l'ameublement des commissions exécutives des agences, des tribunaux et autres établissements publics " : Arch. nat., $\mathrm{F}^{17}$ 1048, "Lettre de la Commission des revenus nationaux à la Commission temporaire des arts réunis au Comité d'Instruction publique », le 3 vendémiaire an III (24 septembre 1794).

28. Voir Arch. nat., $\mathrm{O}^{2} 440,443-452$ et 458. 


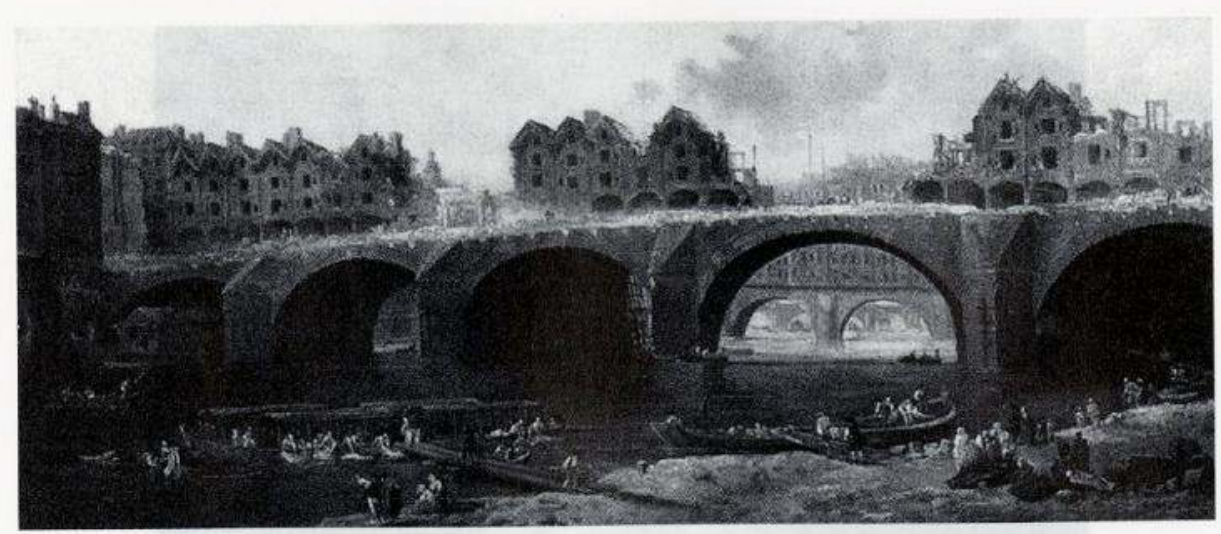

Ill. 2: Démolition du Pont Notre-Dame par Hubert Robert, 1786, H. $73 \mathrm{~cm}$, L. $140 \mathrm{~cm}$ (Musée du Louvre R.F. 1947-38). Ce tableau, dont un double se trouve au musée Carnavalet, fut cédé au ministre des finances et disparut un certain temps sur le marché de l'art. Le Louvre en fit l'acquisition en 1947.

et au palais du Directoire. Si pour la décoration du palais du Luxembourg on privilégie les œuvres flamandes telles que des Van der Meulen, des scènes de genre et des paysages de Mathieu Bril et Karel Dujardin ${ }^{29}$, on remarque que les ministères se réservent surtout des productions françaises récentes: des ouvrages de Lagrenée, Hué, Greuze, Vigée-Lebrun ou Vernet, dont le style commence à être apprécié par les amateurs de la période. C'est ainsi que deux œuvres de Vernet ${ }^{30}$ et un paysage d'Hubert Robert, représentant le temple de Tivoli ${ }^{31}$, gagnent l'hôtel Brissac, siège du ministère de l'intérieur. Ces envois favorisent en effet particulièrement les petits tableaux de paysages et de ruines, agréables et décoratifs : de fait, d'autres compositions d'Hubert Robert ${ }^{32}$ (ill. 2), représentant des paysages parisiens, sont destinées au ministre des finances qui se réserve aussi « deux ruines sur toile genre de Salvator Rosa de Socatelli ${ }^{33}$. Les membres du gouvernement apprécient aussi particulièrement les peintres contemporains, comme l'illustre parfaitement le cas du Paris et Hélène de David (ill. 3), provenant de l'émigré Artois ${ }^{34}$, réservé au dépôt de la rue des Nesle pour le ministre de l'intérieur. Ces tableaux, estimés pour leur manière, sont parfaits pour la décoration, mais leur peu d'ancienneté empêche souvent leur intégration à une collection muséale; ils sont donc placés dans des établissements publics, mais pour la jouissance d'une personne privée ministre ou administrateur. $\mathrm{Si}$ certaines productions, comme ce tableau de David ou l'autoportrait de Madame Vigée-Lebrun et de sa fille ${ }^{35}$ (ill. 4), gagnent le Muséum par la suite, la destinée des biens artistiques placés dans les administrations est particulièrement précaire.

29. Arch. nat., $\mathrm{F}^{17 *} 372-373$, collection Breteuil, $\mathrm{n}^{\circ} 24$ et $\mathrm{n}^{\circ} 43$.

30. Arch. nat., $\mathrm{F}^{17 *} 372-373$, collection de Clermont-Amboise, $\mathrm{n}^{\circ} 6$ et " un clair de lune avec pêcheurs ", collection de l'émigré Dudreneux, $\mathrm{n}^{\circ} 1$.

31. Idem, collection d'Angiviller, $\mathrm{n}^{\circ} 5$.

32. "Deux tableaux représentant la démolition du quai de Grèves et celle du pont au change sur toile de Robert », Arch. nat., $F^{17 *} 372-373$, collection de l'émigré Breteuil, nº 4.

33. Idem, collection Breteuil, $\mathrm{n}^{\circ} 6$.

34. Idem, collection du comte d'Artois.

35. Idem, collection d'Angiviller, $\mathrm{n}^{\circ} 6$. 


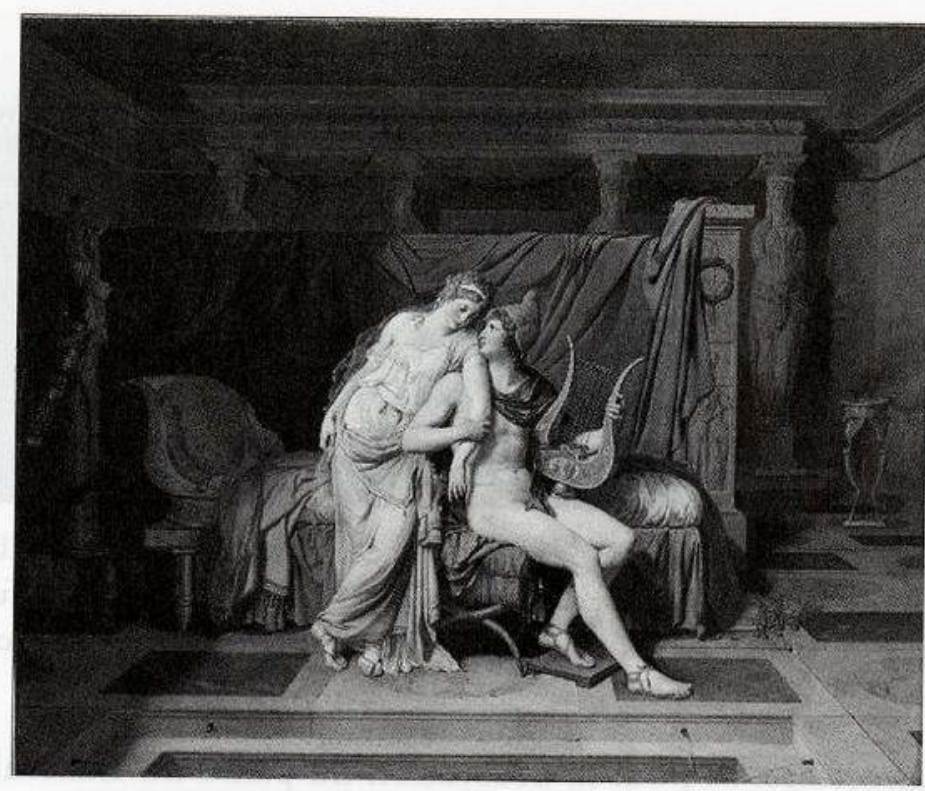

III. 3: Les amours de Paris et Hélène de Jacques-Louis David, 1788, H. $146 \mathrm{~cm}$, L. $181 \mathrm{~cm}$ (Musée du Louvre, Inv. 3696). Cédé au ministre de l'intérieur, puis en dépôt au Luxembourg, ce tableau rejoignit le Louvre en 1823.

Une vraie confusion règne de fait dans tous ces envois, les livraisons se faisant la plupart du temps en masse, sans spécifier la qualité ou la quantité des objets, ce qui facilite grandement les dispersions. Les administrateurs des différents dépôts bénéficient en effet d'une grande liberté pour disposer de ces biens et prennent souvent tout ce qui leur plaît sans réel contrôle; de plus, les ministres suivent l'usage, malgré leur destitution, de conserver les œuvres d'art mises à leur disposition.

Face à ces multiples abus, l'administration des arts commence alors à se poser la question de la restitution des objets après qu'ils ont servi aux ministères, pratique qui ne semble pas encore entrée dans les mœurs. Le ministre Faypoult remarque ainsi, non sans ironie, qu' "une expérience journalière a appris à l'administration du Garde-meuble qu'il était peu d'agents qui se prêtassent de bonne foi à la restitution ${ }^{36}$. En octobre 1797, les commissaires décident donc d'agir contre l'éparpillement des meubles destinés aux administrations: les citoyens Molard et Leblond font un rapport "sur les moyens de conservation des meubles et objets d'art mis à la disposition des divers établissements publics ». Ils remarquent, en premier lieu, que "les objets dont il s'agit appartiennent aux différens établissements où ils ont été placés et non aux chefs et agens de ces établissements " qui ne peuvent être " que des usufruitiers "; dès lors, les objets doivent être " conservés dans leur totalité aux établissements » malgré " les divers mutations qui peuvent ou doivent avoir

36. G. Vauthier, "Le directoire et le Garde-Meuble ", Annales révolutionnaires, Paris, 1914, t. VII, p. 529 . 


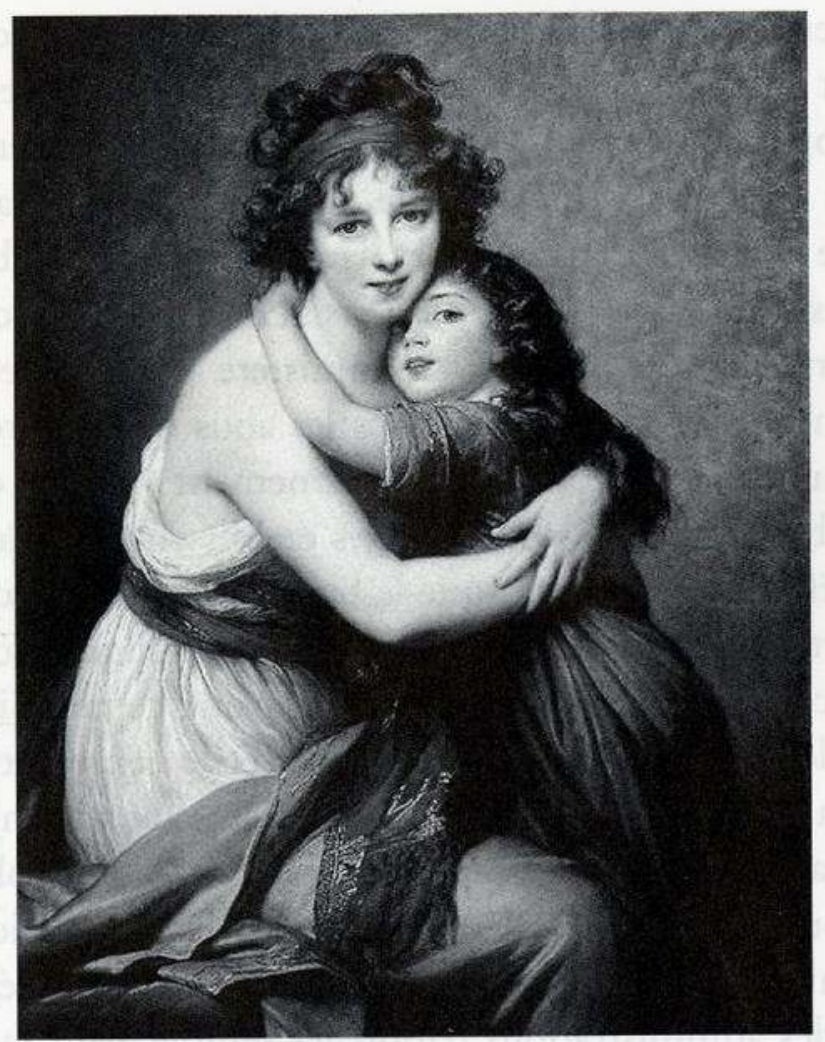

Ill. 4: Madame Vigée-Lebrun et sa fille par Élisabeth-Louise Vigée Le Brun, vers 1789, H. $130 \mathrm{~cm}$, L. $94 \mathrm{~cm}$ (Musée du Louvre, Inv. 3068). Ce tableau, commandé par le comte d'Angiviller en 1789, est réservé pour la décoration de l'Hôtel Brissac, ministère de l'intérieur et rejoint le Muséum du Louvre par la suite.

lieu ${ }^{37}$. Le rapport ajoute que les intéressés doivent « dresser un procès-verbal des objets livrés " et, "à chaque mutation de ministre ou de tout autre agent, le procèsverbal à la main ", faire "la vérification du mobilier attaché à l'établissement " ${ }^{38}$. Pour finir, le rapport incite le Directoire à faire suivre cet arrêté aux administrations concernées ${ }^{39}$. Deux ans plus tard, le Conseil de conservation observe "qu'il n'a été pris jusqu'à ce jour que des mesures insuffisantes pour établir un mode efficace de surveillance à l'égard des objets de sciences et d'arts tirés des dépôts nationaux ", il insiste sur le fait que les "objets mis à la disposition des administrations publiques n'en sont pas moins toujours des propriétés nationales à la Conservation desquelles il est de l'intérêt général de veiller avec soin " ${ }^{40}$.

37. Arch. nat., $\mathrm{F}^{17}$ 1040/B, "Rapport des citoyens Molard et Leblond sur les moyens de conservation des meubles et objets d'art mis à la disposition des divers établissements publics », le 16 vendémiaire an VI (7 octobre 1797).

38. Idem.

39. "Il seroit nécessaire encore que le Directoire prit à cet égard un arrêt qui seroit communiqué à tous ceux qui sont responsables des meubles, livres, tableaux et autres effets qui auroient été mis à leur disposition pour en jouïr à titre d'agent de la République et le successeur de l'Agent qui se seroit retiré auroit déclaré en avoir reçus les effets désignés dans l'état qui lui en auroit été présenté ", idem.

40. Arch. nat., $\mathrm{F}^{17} 1308$, « Procès-verbaux de la Commission temporaire des arts », séance du 11 germinal an VII (31 mars 1799). 
Le gouvernement conçoit donc une réelle administration gouvernementale qui se met petit à petit en place pour gérer le mobilier national. Il est pertinent de voir que ces biens n'appartiennent pas aux agents du gouvernement, mais à l'État et doivent tous, sans exception, passer d'une administration à l'autre. Des chefs de bureau sont même désignés pour assurer, dans chacun des établissements publics, la conservation des objets lors des mutations. Le Conseil de conservation décide également d'établir dans une division du ministère de l'intérieur " un mode uniforme de surveillance propre à prévenir tous les abus qui pourroient se commettre dans les diverses mutations d'agents du Gouvernement " pour " conserver en nature et en totalité les objets dont il s'agit dans les établissements où ils auront été placés ». Toutes ces réflexions montrent que le bureau des musées considère désormais que ces objets d'art placés dans les administrations publiques sont " des propriétés d'État » ${ }^{41}$ et qu'à ce titre, ils peuvent prétendre à une certaine inaliénabilité ou du moins à un contrôle de la part de l'État et à une réelle conservation. L'État cherche bien à empêcher la prescription des œuvres confiées aux administrations.

La période de la Révolution française a donc vu la mise en place d'une administration de l'art pour protéger les œuvres au sein des institutions muséales, mais également les prémices d'une gestion du mobilier national ; l'idée d'un contrôle sur les biens meubles des administrations nationales semble avoir fait son chemin bien avant les décrets sur les listes civiles impériales et les arrêts de jurisprudence du $\mathrm{XIX}^{\mathrm{e}}$ siècle.

\section{Le caractère inviolable de l'objet d'art national}

Toutes ces précautions et la mise en place d'une administration sous entendentt-elles pour autant que l'œuvre d'art nationale s'est vu accorder au cours de la période révolutionnaire un statut d'inaliénabilité telle qu'on le définit aujourd'hui ? L'élaboration d'un droit de garde et de défense désormais dévolu à la Nation n'a certes pas éradiqué tout abus, et l'application d'une certaine forme d'inaliénabilité reste souvent sélective, trop dépendante du pouvoir pour empêcher celui-ci d'agir selon ses exigences. Pourtant, cette collection nationale ne tend pas à devenir un simple cabinet d'amateur. Une fois sélectionnées, les œuvres d'art nationales intègrent ce nouvel espace régi par une administration de l'art et, par cette affectation, revêtent une signification bien plus probante que l'objet en lui-même : elles participent d'une sacralité nouvelle. Dans les faits, les collections de la Couronne, du clergé ou des émigrés étaient composées des mêmes objets qui feront la gloire

41. "Les objets dont il s'agit appartiennent aux différents établissements où ils ont été placés et non aux chefs et agens de ces établissements, de manière que les citoyens ne soient que des usufruitiers, et que, dans les diverses mutations qui peuvent ou doivent avoir lieu, les objets dont il s'agit fussent conservés pour l'usage de leur successeur. ", Arch. nat., F ${ }^{17}$ 1040/B, Rapport sur le mode de surveillance à établir pour la conservation des livres, cartes, tableaux, statues et autres objets appartenant à la nation et mis, conformément aux loix à la disposition de différentes administrations publiques. 
du Muséum central ; la différence n'est donc pas dans l'objet même, mais dans son symbole et l'action juridique que l'on porte à son égard. La pratique du collectionnisme avait déjà permis l'édification d'un régime de propriété spécifique, l'appropriation des biens artistiques par la Nation mène à la conscience d'une plus grande responsabilité et, partant, à des règles inédites de propriété et de transmission.

Les deux conditions constitutives de l'usus publicus sont en effet réunies pour pouvoir définir cet ensemble artistique : " une condition de fait, l'usage matériel de la chose, et une condition de droit, l'affectation préalable et régulière de la chose à l'usage qui en est fait ". L'œuvre d'art participe donc du domaine public puisqu'elle est affectée à l'usage public et qu'elle a été mise concrètement à la disposition des individus. Dans cette optique, les biens culturels, propriétés nationales, sont assimilés à des res publicae, établies pour la jouissance de la collectivité. L'objet d'art, dès l'instant qu'il est envisagé comme un bien d'intérêt général, intègre, dans la logique révolutionnaire, le nouveau patrimoine. Mais peut-on alors le considérer au même titre que les autres biens du domaine public terres, immeubles, rues, cours d'eau ? $\mathrm{Ne}$ renvoie-t-il pas également à une autre réalité, un symbole, une beauté qui lui vaut une protection, non seulement comme bien public, mais aussi pour sa qualité intrinsèque? Car l'œuvre d'art telle qu'elle est perçue par les révolutionnaires dépasse aussi sa simple affectation. Elle relève du sacré en elle-même, hors du champ du droit, par sa seule appartenance au domaine de l'art. L'objet d'art a en effet pour tâche de représenter une manifestation invisible, une vérité cachée, et ces biens sont extra commercium en raison des droits que l'art et l'instruction ont sur eux. Ils deviennent donc des res divini juris en tant qu'objets culturels qui échappent à la propriété ${ }^{42}$. Comme la société révolutionnaire cherche à créer un ensemble artistique qui fait sens, le nouveau patrimoine national est empreint d'une signification, d'une transcendance qui renoue avec le sacré. Bien qu'ayant rejeté la superstition, les révolutionnaires ont conféré à leur patrimoine artistique un caractère dévotionnel. Comme le fait de posséder des reliques inaliénables établissait la légitimité d'un sanctuaire, d'une communauté religieuse, ou même d'une ville comme Venise, la possession de ces œuvres d'art, si fortement estimées par les élites culturelles européennes, garantit à la nouvelle Nation française une sorte de bien-fondé de son entreprise ${ }^{43}$.

Le projet du Muséum national des Arts, véritable enclos sacré, revêt ici toute son importance: Boissy d'Anglas voit dans l'instauration de ce musée un culte au génie ${ }^{44}$ et parle de "temple des arts à côté de celui des lois " 45 . Varon, de son côté, parle de "temples où un sacerdoce doit enseigner le culte des Beaux-Arts " ${ }^{46}$.

42. Gilles Lhuillier, "Les œuvres d'art, res sacrae ?", Revue de la recherche juridique, 1998-2, p. 353.

43. J.-M. Leniaud, Les Archipels du passé : le patrimoine et son histoire, Paris, Fayard, 2002, p. 41.

44. "Le génie qui doit présider à un établissement consacré uniquement à son culte, réclame tous les secours et toutes les dépenses " : François-Antoine Boissy d'Anglas, Essai sur les fêtes nationales, suivi de quelques idées sur les Arts et sur la nécessité de les encourager, Paris, Imprimerie polyglotte, 1794, p. 29.

45. Ibid., p. 40.

46. Varon, "Rapport du Conservatoire du Muséum national au Comité d'Instruction publique ", cité par Y. Cantarel-Besson, La Naissance du musée du Louvre, t. II, p. 227. 
Afin de concevoir une réelle protection, les commissaires - artistes se doivent de recréer un temple ou tout au moins une aura, à l'intérieur desquels les biens pourraient bénéficier d'une situation à part. S'appuyant sur le développement juridique du statut de l'œuvre d'art, l'administration du Muséum durcit d'ailleurs ses positions sous le Directoire : en février 1797, alors qu'un artiste demande au Conseil du Muséum de lui prêter un tableau, certainement pour en faire un copie, l'administration refuse et arrête aussitôt qu' " aucun tableau, carton, dessin, bronze, vases, et généralement aucun des objets d'arts déposés dans le Musée central des Arts ne pourra être prêté ou communiqué à qui que ce soit, hors de l'enceinte du Musée ». Pour se justifier, ils assurent qu'une telle décision n'est pas de leur ressort affirmant que "l'administration du Musée central des Arts s'interdit [...] à elle-même la faculté de délibérer sur aucune demande de cette nature, sauf invitation du Directoire exécutif $" ~ "{ }^{47}$. Le message est limpide : il n'est pas du seul ressort de l'administration du Musée de statuer sur les œuvres nationales, mais ils doivent désormais en référer aux hautes instances de l'État pour décider du sort des objets qui ont intégré l'enceinte sacrée du Muséum. Les révolutionnaires établissent même un contrôle sur tout ce qui sort de cet établissement, soumettant tout accord à l'aval du Directoire exécutif et au laissez-passer des représentants de la Nation.

Les œuvres d'art, intégrées aux collections muséales, seraient donc les héritières des res sacrae antiques et d'un régime de conservation transmis à travers les siècles par l'Église. La protection et l'inaliénabilité relative appliquées aux œuvres d'art nationales découleraient de leur consécration à l'Instruction publique de tous les citoyens, mais dépendraient aussi de ces valeurs inhérentes aux œuvres qui ont permis leur sélection. La valeur intrinsèque du bien artistique transcende toujours l'idée d'une simple affectation d'un bien à un domaine public. Après avoir permis ce tri, la société révolutionnaire se devait d'établir un ensemble stable, inébranlable, libéré des jugements personnels, pour devenir ce musée qui vise à « la connaissance par-delà les vicissitudes du temps, du goût et des appréciations " ${ }^{48}$. Il faut tout même voir que ce principe d'inaliénabilité est aisément mis de côté sous le Consulat et l'Empire quand Bonaparte se permet de s'approprier des chefs-d'œuvre pour son usage personnel, même si la propriété publique des œuvres d'art nationalisées demeure ancrée dans les esprits. En ce qui concerne les restitutions aux familles de condamnés et de certains émigrés des biens qui leur ont été séquestrés, la politique suivie est alors majoritairement la sauvegarde de la propriété nationale. Le 22 thermidor an VIII (10 août 1800), le ministre de l'intérieur, Lucien Bonaparte, prévient les administrations, qu'elles ne doivent accepter aucune cession d'œuvres d'art aux anciens émigrés :

47. Y. Cantarel-Besson, Musée du Louvre, p. 31, séance du 15 pluviôse an V (3 février 1797).

48. Dominique Schnapper, "Des collections anatomiques aux objets de culte : conservation et exposition des restes humains dans les musées ", Symposium international du quai Branly, les 22 et 23 février 2008, p. 91. 
La nécessité de prévenir le dépouillement absolu des bibliothèques et des musées [...] m'a déterminé à arrêter qu'aucun objet des sciences et d'arts déposés dans ces établissements ne serait remis aux personnes qui, après radiation de la liste des émigrés ou leur rappel, sont rentrées en possession de leurs autres biens ${ }^{49}$.

L'administration des arts veut formellement mettre fin aux " réclamations que l'intérêt de l'Instruction publique doit écarter ». Le ministre de l'intérieur tient en effet à garder l'acquis de la Révolution et si les œuvres encore en dépôt peuvent être remises au fondé de pouvoir de certaines familles, les biens affectés aux musées doivent être préservés en tant que propriété nationale. Ces biens participant du domaine artistique ont donc acquis un nouveau statut au sein d'institutions reconnues d'intérêt général, statut confirmé ensuite par le gouvernement de la Restauration qui admet que : " D'après les règles établies, tout objet d'art et de science porté sur un catalogue ou inscrit sur un registre a été censé aliéné et n'a point été jugé dans le cas d'être rendu, se trouvant ainsi affecté à un service public ${ }^{50}$ ". De nombreuses réclamations sont ainsi déboutées car désormais les objets d'arts participent des "établissements publics ${ }^{51}$ ". Le concept d'inaliénabilité des collections françaises s'est donc inscrit nationalement au cours de la Révolution; il faut attendre l'Empire pour qu'il soit entériné par un décret, mais la réalité d'une protection juridique d'un patrimoine qui devait conduire à la régénération de toute la société est déjà amorcée avec la politique patrimoniale mise en place au cours de ces vingt années fondatrices.

Finalement, le destin des biens publics sous la Révolution a été de "la compétence du législateur ${ }^{52}$ ": que le domaine soit inaliénable tout en pouvant être vendu par décret, ou qu'il soit aliénable sauf certaines exceptions permises par la loi, il reste que la Nation reconnaît à certains biens la faculté de ne pouvoir être cédés selon la loi du marché. Par une mesure spéciale, l'homme de loi peut donc attribuer à une catégorie de biens un statut proche de l'inaliénabilité en les affectant $\mathrm{au}$ "bien public» et permettre alors leur conservation. Si les décrets révolutionnaires montrent les contradictions d'une société qui cherche encore à se définir, la réalité des faits confirme l'existence de biens qui bénéficient d'un régime de propriété exceptionnel. Les événements de la Révolution donnent lieu à une définition d'un espace de l'art quasi sacré, conférant aux œuvres qui le composent, si ce n'est une inaliénabilité juridique, du moins une inaliénabilité de fait. Malgré l'idée de dispersion et division de fonds qu'ont entraînée les nationalisations, les révolutionnaires cherchent à former un patrimoine intègre. Cet ensemble qu'ils tentent d'édi-

49. Arch. dép. Rhône, T. 384, cité par Annie Héritier, Genèse du patrimoine artistique. Élaboration d'une notion juridique. 1750-1816, Paris, l'Harmattan, 2003, p. 241.

50. Arch. nat., $\mathrm{F}^{21} 574$, " Note du ministère de l'intérieur ", le 9 août 1814.

51. Idem.

52. Hubert Hubrecht, "L'inaliénabilité. Passé et avenir d'un principe de droit constitutionnel ", La Constitution et ses valeurs. Mélanges en l'honneur de Dmitri Georges Lavroff, Paris, Dalloz, 2005, p. 421. 
fier sur des bases juridiques tend donc à l'unicité, même s'il n'est pas strictement défini par des interdits. Outre les dispositions légales, il faut considérer l'opinion publique qui a pleinement adopté ce patrimoine intrinsèquement lié au territoire français. Le désir de transparence et de publicité a en effet projeté sur les devants de la scène les attentes et espoirs des citoyens français. La conscience d'une propriété collective et nationale est l'un des apports les plus manifestes de la Révolution : la société cherchant dans ce patrimoine culturel à s'identifier par rapport à un passé qu'on préserve pour les générations futures.

\author{
Olivia BRISSAUD \\ archiviste paléographe
}

